\title{
Changes in the MicroRNA Profile Observed in the Subcutaneous Adipose Tissue of Obese Patients after Laparoscopic Adjustable Gastric Banding
}

\author{
Carmela Nardelli, ${ }^{1,2}$ Laura Iaffaldano, ${ }^{1,2}$ Vincenzo Pilone, ${ }^{3}$ Giuseppe Labruna, ${ }^{4}$ \\ Maddalena Ferrigno, ${ }^{2}$ Nicola Carlomagno, ${ }^{5}$ Concetta Anna Dodaro, ${ }^{5}$ Pietro Forestieri, ${ }^{6}$ \\ Pasqualina Buono, ${ }^{4,7}$ Francesco Salvatore, ${ }^{1,2}$ and Lucia Sacchetti ${ }^{2}$ \\ ${ }^{1}$ Dipartimento di Medicina Molecolare e Biotecnologie Mediche, Università di Napoli Federico II, Via S. Pansini 5, 80131 Naples, Italy \\ ${ }^{2}$ CEINGE-Biotecnologie Avanzate Scarl, Via G. Salvatore 486, 80145 Naples, Italy \\ ${ }^{3}$ Dipartimento di Medicina e Chirurgia, Università di Salerno, Via Giovanni Paolo II 132, Fisciano, 84084 Salerno, Italy \\ ${ }^{4}$ IRCCS SDN-Istituto di Ricerca Diagnostica e Nucleare, Via Gianturco 113, 80100 Naples, Italy \\ ${ }^{5}$ Dipartimento di Scienze Biomediche Avanzate, Università di Napoli Federico II, Via S. Pansini 5, 80131 Naples, Italy \\ ${ }^{6}$ Dipartimento di Medicina Clinica e Chirurgia, Università di Napoli Federico II, Via S. Pansini 5, 80131 Naples, Italy \\ ${ }^{7}$ Dipartimento Scienze Motorie e del Benessere, Università di Napoli Parthenope, Via Amm. F. Acton 38, 80133 Naples, Italy
}

Correspondence should be addressed to Lucia Sacchetti; sacchett@unina.it

Received 28 December 2016; Revised 17 February 2017; Accepted 23 February 2017; Published 13 March 2017

Academic Editor: R. Prager

Copyright (c) 2017 Carmela Nardelli et al. This is an open access article distributed under the Creative Commons Attribution License, which permits unrestricted use, distribution, and reproduction in any medium, provided the original work is properly cited.

Background. Laparoscopic adjustable gastric banding (LAGB) results in significant lasting weight loss and improved metabolism in obese patients. To evaluate whether epigenetic factors could concur to these benefits, we investigated the subcutaneous adipose tissue (SAT) microRNA (miRNA) profile before (T0) and three years (T1) after LAGB in three morbidly obese women. Case Reports. SAT miRNA profiling, evaluated by TaqMan Array, showed four downexpressed (miR-519d, miR-299-5p, miR-212, and miR-671-3p) and two upexpressed (miR-370 and miR-487a) miRNAs at T1 versus T0. Bioinformatics predicted that these miRNAs regulate genes belonging to pathways associated with the cytoskeleton, inflammation, and metabolism. Western blot analysis showed that PPAR-alpha, which is the target gene of miR-519d, increased after LAGB, thereby suggesting an improvement in SAT lipid metabolism. Accordingly, the number and diameter of adipocytes were significantly higher and lower, respectively, at T1 versus T0. Bioinformatics predicted that the decreased levels of miR-212, miR-299-5p, and miR-671-3p at T1 concur in reducing SAT inflammation. Conclusion. We show that the miRNA profile changes after LAGB. This finding, although obtained in only three cases, suggests that this epigenetic mechanism, by regulating the expression of genes involved in inflammation and lipid metabolism, could concur to improve SAT functionality in postoperative obese patients.

\section{Introduction}

Obesity is a major health problem worldwide [1]. Besides lifestyle interventions and pharmacotherapy, significant, lasting weight loss in obese patients can be obtained with laparoscopic adjustable gastric binding (LAGB) surgery $[2,3]$. This procedure is usually applied in morbidly obese patients (BMI greater than $40 \mathrm{~kg} / \mathrm{m}^{2}$ ) and/or if obesity is associated with comorbidities, such as diabetes or cardiovascular diseases [4]. We previously reported that LAGB also improved metabolism, in terms of inflammation, insulin resistance, and liver steatosis, three years after LAGB [3]. MicroRNAs (miRNAs) are short (19-22 bp) noncoding RNAs that regulate the expression of mRNA targets mainly by binding their complementary sequences at the $3^{\prime}$ untranslated region ( $3^{\prime}$ UTR) and inhibiting their translation [5]. The miRNA profile in subcutaneous (SAT) and in visceral (VAT) adipose tissues differs between obese and lean subjects $[6,7]$. The aim of the present study was to investigate the SAT miRNA profile in 
three morbidly obese women before (T0) and three years (T1) after LAGB and to evaluate whether miRNAs are involved in post-LAGB metabolic improvement.

\section{Methods}

2.1. Patients. Three obese women (mean body mass index [BMI] $42.9 \mathrm{~kg} / \mathrm{m}^{2}$; mean age 48 years) undergoing LAGB (at $\mathrm{T} 0$ and $\mathrm{T} 1$ ) and two lean controls (mean BMI $21.5 \mathrm{~kg} / \mathrm{m}^{2}$; mean age 37 years) undergoing laparoscopic cholecystectomy entered the study. All subjects gave informed consent to the study, which was performed according to the Helsinki II Declaration and was approved by the Ethics Committee of our School of Medicine.

2.2. Serum Determinations. A fasted serum sample was also collected from enrolled subjects, the main biochemical parameters were measured by routine methods; leptin (L) and adiponectin (A) adipokines were measured by ELISA methods and the L/A ratio was calculated.

2.3. Adipocytes Size and Number Quantification. Periumbilical bioptic SAT samples were obtained from all subjects. All biopsies were snap-frozen and stored in liquid nitrogen until RNA isolation. The number and diameter of adipocytes (at T0 and T1) were measured as previously reported [3]. Briefly, hematoxylin and eosin stains were used to identify the cellular components and their morphological changes. For each sample, adipocytes were counted in three fields $(100 \mu \mathrm{m}$ each) and their size was measured by two operators; the average of each parameter was then calculated.

2.4. RNA Isolation. Total RNA (including miRNAs) was purified from SAT using the mirVana ${ }^{\mathrm{TM}}$ miRNA isolation kit (Ambion, Austin, TX, USA), and its concentration was evaluated by NanoDrop ${ }^{\circledR}$ ND-1000 UV-Vis spectrophotometer (NanoDrop Technologies, Wilmington, DE, USA).

2.5. miRNAs Expression Profile. TaqMan low density arrays Human MicroRNA Panel v1.0 (Applied Biosystems Inc., Foster City, CA, USA), containing 377 preloaded human miRNA targets and the endogenous control RNU48, were used according to the manufacturer's instructions. RT-PCR was performed with $800 \mathrm{ng}$ of cDNA and the $7900 \mathrm{HT}$ real-time PCR system (Applied Biosystems) as reported elsewhere [7]. miRNA expression was normalized to RNU48 and quantified using the RQ Manager 1.2 software (Applied Biosystems) with the following formula: $\mathrm{RQ}=2^{-\Delta \Delta \mathrm{Ct}}$, where $\Delta \Delta \mathrm{Ct}=$ $\left(\mathrm{Ct}_{\text {obese }}[\mathrm{miRNA}]-\mathrm{Ct}_{\text {obese }}[\mathrm{RNU} 48]\right)-\left(\mathrm{Ct}_{\text {calibrator }}[\mathrm{miRNA}]-\right.$ $\mathrm{Ct}_{\text {calibrator }}$ [RNU48]). miRNAs whose mean baseline RQ levels were $<0.5$ (downexpressed) or $>2.0$ (upexpressed) in all obese patients versus controls were considered differently expressed. We compared the expression of these differently expressed miRNAs with those previously reported in the SAT of other obese cohorts [6, 8-14]. Only miRNAs whose expression trend was confirmed were selected for evaluation after LAGB.

The levels of miR-370 and miR-487a (upexpressed) and miR-519d (downexpressed) were validated by TaqMan
miRNA assays (Applied Biosystems) in accordance with the manufacturer's instructions on the $7900 \mathrm{HT}$ real-time PCR system (Applied Biosystems).

2.6. PPAR-Alpha Western Blot. Total proteins were extracted, quantified, and separated by $15 \%$ sodium dodecyl sulfatepolyacrylamide gel electrophoresis. Western blot analyses of PPAR- $\alpha$ (dilution 1:400) and actin (dilution 1:1000) proteins were performed with antibodies from Santa Cruz Biotechnology (Santa Cruz, CA, USA) [6].

2.7. Bioinformatics. The MiRTarBase, STRING, and KEGG databases were used to select the experimentally validated miRNA/target gene interaction, to explore protein-protein interactions and to identify the pathways significantly $(p<$ 0.001) deregulated, respectively.

2.8. Statistics. Biochemical and clinical data are expressed as mean and standard error of the mean (SEM), whereas miRNA expression data are reported as $\log _{10}$ mean RQ and SEM. The Wilcoxon test was used to compare data obtained at T0 and T1. Differences were considered statistically significant at $p<0.05$. Statistical analyses were carried out with the PASW Statistics (Ver.18; SPSS Inc. Headquarters, Chicago, Ill. USA).

\section{Results}

Leptin levels and the L/A ratio were lower, whereas adiponectin levels were higher, although not significantly, at T1 versus T0 (Table 1). Furthermore, the number of SAT adipocytes was higher and their diameter lower $(p<0.05)$ at T1 versus T0 (Table 1) in all three obese patients. Overall, $68 \%$ (257/377) of miRNAs were expressed at T0 in SAT from obese and control subjects. Most of these miRNAs (199/257) were not differentially expressed between obese and lean subjects and were not further investigated, whereas $58 \mathrm{miRNAs}$ were upexpressed $(74 \%, 43 / 58)$ or downexpressed $(26 \%, 15 / 58)$ in obese versus controls (Table 2 ).

Table 2 also shows the 31/58 miRNAs (25 upexpressed and 6 downexpressed) previously reported to have a similar obese-associated trend in other cohorts [6, 8-14]. Six of these 31 miRNAs showed post-LAGB changes: miR-519d, miR299-5p, miR-212, and miR-671-3p were downexpressed at T1 versus $\mathrm{T} 0$, and $\mathrm{miR}-370$ and $\mathrm{miR}-487 \mathrm{a}$ were upexpressed at $\mathrm{T} 1$ versus T0 (Figure 1(a)). Single RT-PCR assay confirmed miR370, miR-487a, and miR-519d post-LAGB expression (mean RQ values: 3.54, 3.79, and 0.65, resp., at T1 versus T0). Notably, at Western blot analysis, the expression of protein PPAR- $\alpha$, whose mRNA is targeted by miR-519d [6], was significantly higher $(p<0.05)$ at T1 versus T0 (mean OD/SD: $1.84 / 0.3$ versus $0.72 / 0.4$, resp.) (Figure $1(\mathrm{~b})$ ).

Bioinformatics predicted that, by targeting several genes, these six miRNAs significantly $(p<0.001)$ regulated interrelated pathways associated with the cytoskeleton, inflammation, and metabolism, that is, FoxO, cell cycle, toll-like receptor, MAPK, AMPK, p53, PI3K-Akt, adipocytokine, tight and adherens junctions, focal adhesion, mTOR, PPAR, TGFbeta, and apoptosis (Table 3 and Figure 2). 
TABLE 1: Clinical and biochemical parameters measured in three obese females (OB) before (T0) and three years after LAGB (T1).*

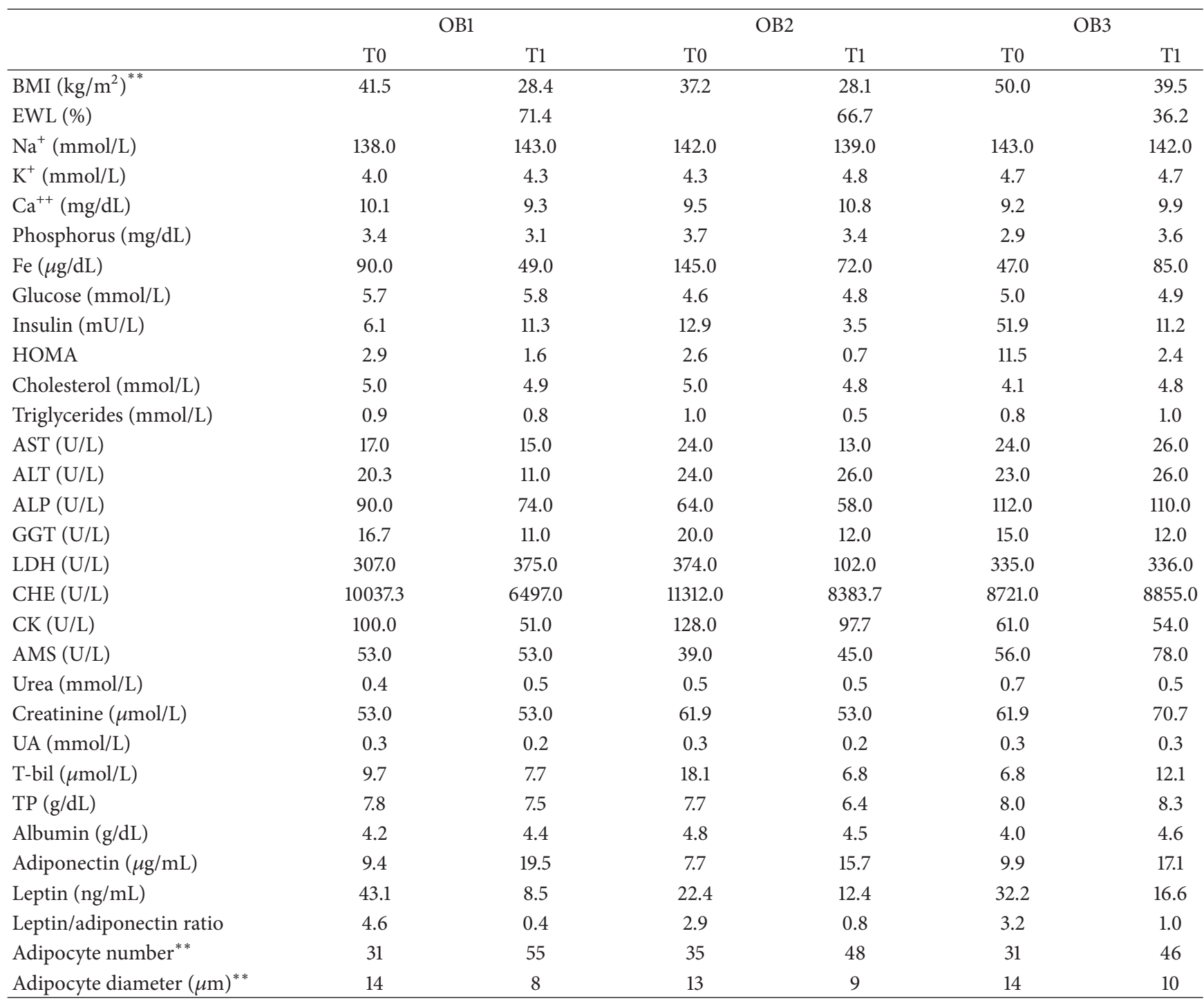

${ }^{*}$ Data from [3]. ${ }^{* *}$ Statistically significant differences at T0 versus $\mathrm{T} 1$ at Wilcoxon's test; $p<0.01$.

ALP: alkaline phosphatase; ALT: alanine aminotransferase; AMS: amylase; AST: aspartate aminotransferase; BMI: body mass index; CHE: cholinesterase; CK: creatine kinase; EWL: excess weight loss; GGT: $\gamma$-glutamyl transferase; HOMA: homeostasis model assessment; LDH: lactate dehydrogenase; T-bil: total bilirubin; TP: total proteins; UA: uric acid.

\section{Discussion}

In three obese patients, we identified 6 miRNAs whose expression levels were either lower (miR-519d, miR-212, miR299-5p, and -miR-671-3p) or higher (miR-370 and miRNA487a) after LAGB. These miRNAs were predicted to regulate pathways involved in obesity or in obesity-related cellular dysfunctions, the most significant being adipocytokine and PPAR signaling, some interrelated metabolic pathways (FoxO, cell cycle, MAPK, AMPK, p53, PI3K-Akt, TGFbeta signaling, and apoptosis), the cytoskeleton, and cell-cell adhesion (focal adhesion and tight and adherens junctions).

We previously found higher miR-519d expression in SAT from another group of obese patients versus lean subjects and demonstrated that it targeted PPAR- $\alpha$ mRNA, thereby reducing the level of this protein in SAT and causing lipid accumulation during adipocyte differentiation [6]. Data obtained in PPAR- $\alpha$-null mice showed that this protein is involved in lipid homeostasis [15]. Thus, our finding of lower miR-519d levels and higher PPAR- $\alpha$ protein expression in SAT after LAGB, together with the significantly $(p<$ 0.05 ) smaller diameter and higher number of adipocytes at T1 versus T0, suggests that surgery might improve lipid metabolism and adipose tissue functionality also via a miR519d-mediated mechanism. Accordingly, PPAR- $\alpha$ activation was reported to induce removal of intracellular lipids through fatty acid oxidation [15] and to be a part of a regulatory loop that controls the metabolic response of adipose tissue to nutrients and other signals [15]. Furthermore, PPAR- $\alpha$ is also a nutritional sensor adapting metabolic homeostasis to 
TABLE 2: Differently expressed miRNAs at T0 in obese patients versus normal-weight subjects.

\begin{tabular}{|c|c|}
\hline Upexpressed miRNAs & Downexpressed miRNAs \\
\hline hsa-let-7a & hsa-miR-18b \\
\hline hsa-let-7d & hsa-miR-107 \\
\hline hsa-let-7e & $h s a-m i R-200 a$ \\
\hline hsa-let-7f & $h s a-m i R-200 c$ \\
\hline$h s a-m i R-10 a$ & hsa-miR-205 \\
\hline$h s a-m i R-21$ & hsa-miR-331-5p \\
\hline$h s a-m i R-27 b$ & hsa-miR-342-5p \\
\hline$h s a-m i R-29 c$ & hsa-miR-370 \\
\hline hsa-miR-34a & hsa-miR-382 \\
\hline$h s a-m i R-92 a$ & hsa-miR-429 \\
\hline hsa-miR-95 & $h s a-m i R-487 a$ \\
\hline$h s a-m i R-99 b$ & hsa-miR-496 \\
\hline hsa-miR-100 & hsa-miR-520a-3p \\
\hline$h s a-m i R-125 b$ & hsa-miR-548c-5p \\
\hline hsa-miR-128 & hsa-miR-615-5p \\
\hline \multicolumn{2}{|l|}{ hsa-miR-130a } \\
\hline \multicolumn{2}{|l|}{ hsa-miR-130b } \\
\hline \multicolumn{2}{|l|}{ hsa-miR-145 } \\
\hline \multicolumn{2}{|l|}{$h s a-m i R-146 b-5 p$} \\
\hline \multicolumn{2}{|l|}{ hsa-miR-190 } \\
\hline \multicolumn{2}{|l|}{ hsa-miR-195 } \\
\hline \multicolumn{2}{|l|}{ hsa-miR-199b-5p } \\
\hline \multicolumn{2}{|l|}{ hsa-miR-204 } \\
\hline \multicolumn{2}{|l|}{$h s a-m i R-212$} \\
\hline \multicolumn{2}{|l|}{ hsa-miR-221 } \\
\hline \multicolumn{2}{|l|}{ hsa-miR-224 } \\
\hline \multicolumn{2}{|l|}{ hsa-miR-296-5p } \\
\hline \multicolumn{2}{|l|}{$h s a-m i R-299-5 p$} \\
\hline \multicolumn{2}{|l|}{ hsa-miR-323-3p } \\
\hline \multicolumn{2}{|l|}{ hsa-miR-328 } \\
\hline \multicolumn{2}{|l|}{ hsa-miR-339-5p } \\
\hline \multicolumn{2}{|l|}{ hsa-miR-362-3p } \\
\hline \multicolumn{2}{|l|}{$h s a-m i R-365$} \\
\hline \multicolumn{2}{|l|}{ hsa-miR-494 } \\
\hline \multicolumn{2}{|l|}{ hsa-miR-519d } \\
\hline \multicolumn{2}{|l|}{ hsa-miR-520g } \\
\hline \multicolumn{2}{|l|}{ hsa-miR-532-3p } \\
\hline \multicolumn{2}{|l|}{$h s a-m i R-652$} \\
\hline \multicolumn{2}{|l|}{ hsa-miR-655 } \\
\hline \multicolumn{2}{|l|}{ hsa-miR-671-3p } \\
\hline \multicolumn{2}{|l|}{ hsa-miR-708 } \\
\hline hsa-miR-744 & \\
\hline hsa-miR-758 & \\
\hline
\end{tabular}

Italic type indicates miRNAs whose expression trend was similar to those of previous studies [6, 8-14]. These miRNAs were investigated after LAGB.

energy deprivation; it exerts an anti-inflammatory function and controls also some aspects of the glucose and lipid metabolism in several tissues included the adipose one [16].

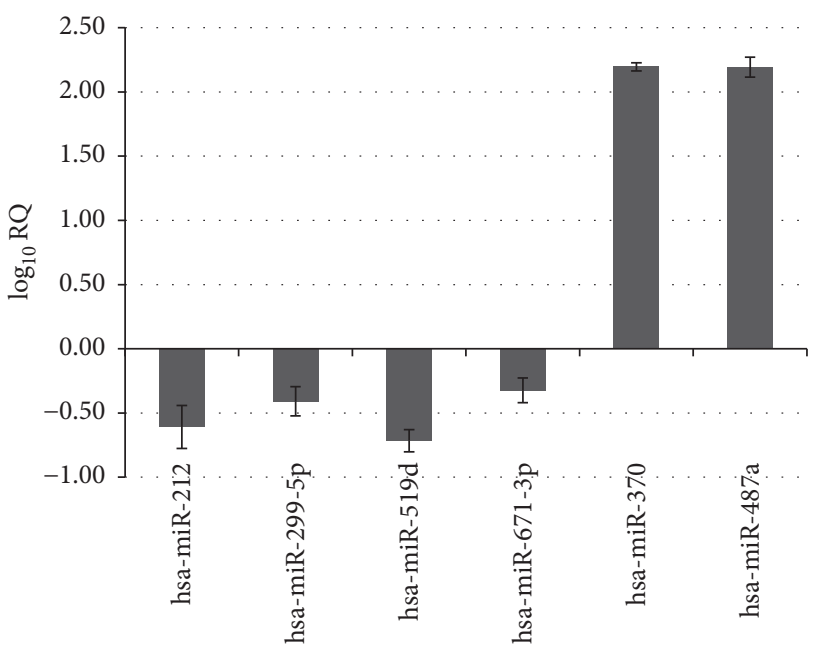

(a)

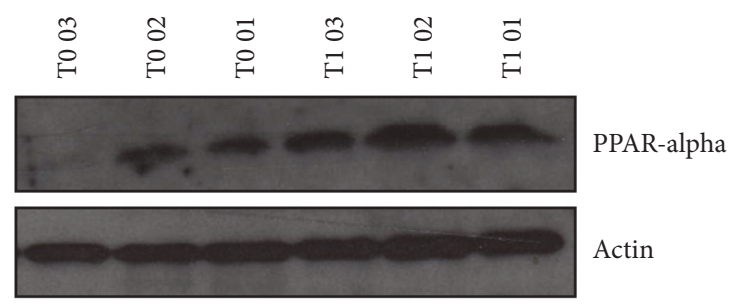

(b)

FIgURE 1: Six miRNAs whose expression changed in subcutaneous adipose tissue (SAT) three years after LAGB (T1) and Western blot evaluation of protein $\operatorname{PPAR} \alpha$, which is targeted by miR-519d. (a) Expression levels of 4 downexpressed (miR212, miR-299-5p, miR-519d, and miR-671-3p) and 2 upexpressed miRNAs (miR-370 and miR-487a) after LAGB (T1 versus T0). MiRNA expression levels are shown as mean $\log _{10}$ RQ (the miRNA expression values were first normalized to RNU48, after which the relative quantification was calculated as RQ = $2^{-\Delta \Delta \mathrm{Ct}}$, where $\Delta \Delta \mathrm{Ct}=[\mathrm{Ct}$ obese $($ miRNA $)-\mathrm{Ct}$ obese $(\mathrm{RNU} 48)]-$ [Ct calibrator(miRNA)-Ct calibrator(RNU48)]. (b) Western blot evaluation of protein PPAR- $\alpha$ from SAT of 3 obese patients before (T0) and three years (T1) after LAGB.

The decrease of miR-212, miR-299-5p, and miR-671-3p levels that we found at $\mathrm{T} 1$ versus $\mathrm{T} 0$ was predicted to be associated with reduced SAT inflammation. This is in line with the findings that (i) miR-212 is directly involved in inflammation and immune processes [17] and (ii) miR-2995 p, by upregulating its target gene SPP1, could promote endocytosis of cell debris and indirectly reduce inflammation [18]. Lastly, an earlier study showed that miR-671-3p expression was lower in visceral fat from obese patients with nonalcoholic steatohepatitis versus obese patients with nonalcoholic fatty liver disease [19] and was related to decreased liver inflammation.

Concerning miR-212, it was demonstrated to be upexpressed in high fat fed mice and its levels measured in liver were downregulated by physical activity [20]. miR212 positively regulated fatty acids biosynthesis and hepatic storage by acting on fibroblast growth factor 21 (FGF-21) 
TABLE 3: Metabolic pathways predicted to change by our miRNAs after LAGB. The most significant pathways $(p<0.005)$ predicted by KEGG to contain genes targeted by the 6 miRNAs whose expression changed three years after LAGB.

\begin{tabular}{|c|c|c|}
\hline \multicolumn{3}{|c|}{ KEGG pathways } \\
\hline Pathway description (ID) & FDR & miRNAs \\
\hline $\begin{array}{l}\text { FoxO signaling pathway (4068) } \\
\text { AKT3, CCNB1, CDKN1A, FOXO1, PTEN, SOD2, } \\
\text { TGFBR2 }\end{array}$ & $1.05 e-07$ & $\begin{array}{l}\text { miR-519d, miR-212, } \\
\text { miR-299-5p, miR-370 }\end{array}$ \\
\hline $\begin{array}{l}\text { Cell cycle (4110) } \\
\text { CCNA2, CCNB1, CDKN1A, MYC, RB1 }\end{array}$ & $4.03 e-05$ & $\begin{array}{l}\text { miR-519d, miR-299-5p, } \\
\text { miR-212 }\end{array}$ \\
\hline $\begin{array}{l}\text { Toll-like receptor signaling pathway (4620) } \\
\text { AKT3, IRAK4, MAP3K8, SPP1 }\end{array}$ & 0.000363 & $\begin{array}{l}\text { miR-519d, miR-299-5p, } \\
\text { miR-212, miR-370 }\end{array}$ \\
\hline $\begin{array}{l}\text { MAPK signaling pathway (4010) } \\
\text { AKT3, MAP3K8, MYC, NF1, TGFBR2 }\end{array}$ & 0.000567 & $\begin{array}{l}\text { miR-519d, miR-212, } \\
\text { miR-370 }\end{array}$ \\
\hline $\begin{array}{l}\text { AMPK signaling pathway (4152) } \\
\text { AKT3, CCNA2, CPT1A, FOXO1 }\end{array}$ & 0.000567 & $\begin{array}{l}\text { miR-519d, miR-212, } \\
\text { miR-370 }\end{array}$ \\
\hline $\begin{array}{l}\text { p53 signaling pathway (4115) } \\
\text { CCNB1, CDKN1A, PTEN }\end{array}$ & 0.00167 & $\begin{array}{l}\text { miR-519d, miR-299-5p, } \\
\text { miR-212 }\end{array}$ \\
\hline $\begin{array}{l}\text { PI3K-Akt signaling pathway (4151) } \\
\text { AKT3, CDKN1A, MYC, PTEN, SPP1 }\end{array}$ & 0.00167 & $\begin{array}{l}\text { miR-519d, miR-299-5p, } \\
\text { miR-212 }\end{array}$ \\
\hline $\begin{array}{l}\text { Adipocytokine signaling pathway (4920) } \\
\text { AKT3, CPT1A, PPARA }\end{array}$ & 0.00167 & miR-519d, miR-370 \\
\hline $\begin{array}{l}\text { Tight junction (4530) } \\
\text { AKT3, PTEN, TJP1 }\end{array}$ & 0.00821 & miR-519d, miR-212 \\
\hline $\begin{array}{l}\text { Focal adhesion (4510) } \\
\text { AKT3, PTEN, SPP1 }\end{array}$ & 0.0259 & miR-519d, miR-299-5p \\
\hline $\begin{array}{l}\text { mTOR signaling pathway (4150) } \\
\text { AKT3, PTEN }\end{array}$ & 0.0269 & miR-519d \\
\hline $\begin{array}{l}\text { PPAR signaling pathway (3320) } \\
\text { CPT1A, PPARA }\end{array}$ & 0.0346 & miR-370, miR-519d \\
\hline $\begin{array}{l}\text { Adherens junction (4520) } \\
\text { TGFBR2, TJP1 }\end{array}$ & 0.0356 & miR-370, miR-212 \\
\hline $\begin{array}{l}\text { TGF-beta signaling pathway ( } 4350) \\
\text { MYC, TGFBR2 }\end{array}$ & 0.0418 & miR-212, miR-370 \\
\hline $\begin{array}{l}\text { Apoptosis }(4210) \\
\text { AKT3, IRAK4 }\end{array}$ & 0.0483 & miR-519d, miR-212 \\
\hline
\end{tabular}

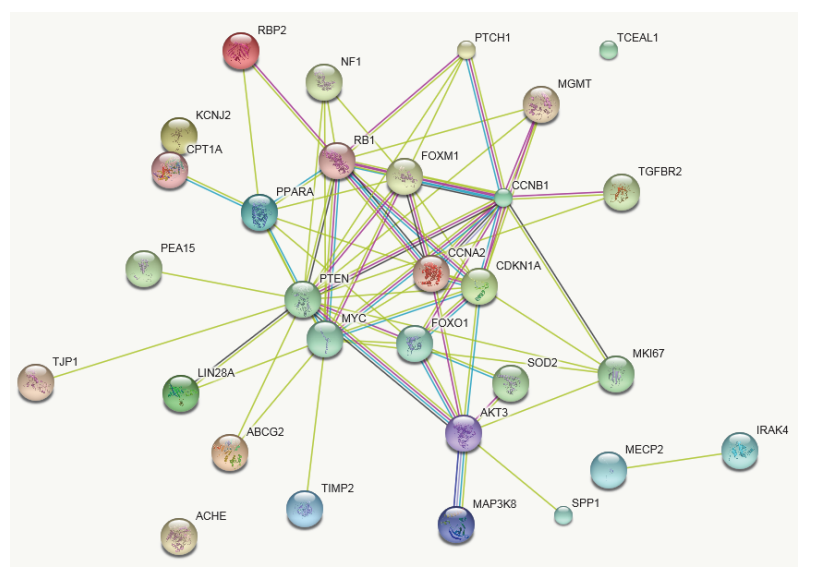

FIGURE 2: Metabolic pathways predicted to change by our miRNAs after LAGB. Network identified by STRING showing a close interaction among the proteins regulated by the subset of 6 miRNAs. The above 6 miRNAs also targeted the pathways of several proteins in cancer and infection diseases (data not reported).
[20]. Interestingly, FGF-21 is a target gene of PPAR- $\alpha$ and is involved in cellular response to oxidative stress [21].

Consequently, the reduced SAT expression of both miR212 and $-519 \mathrm{~d}$, that we observed in our obese patients after LAGB, could exert a role in reducing oxidative stress and inflammation by acting on the PPAR- $\alpha$-FGF- 21 axis. In agreement with this hypothesis, we previously reported a reduced SAT inflammation after LAGB in a larger cohort compared to the present [3] and here we suggest that this effect could be, in part, caused by a miRNA-mediated mechanism.

Finally, although miR-370 and 487a, which were upexpressed after LAGB, were predicted to regulate the expression of genes belonging to several metabolic pathways, their contribution to surgery metabolic improvement, if any, remains to be established.

\section{Conclusions}

In conclusion, although our data were obtained in only three patients (mainly because patients were unwilling to 
undergo a post-LAGB biopsy), we show that the miRNA profile changes after LAGB. This finding suggests that this epigenetic mechanism, by regulating the expression of genes involved in inflammation and lipid metabolism, could concur to improve SAT functionality in postoperative obese patients.

\section{Conflicts of Interest}

The authors declare no conflicts of interest regarding the publication of this paper.

\section{Authors' Contributions}

Carmela Nardelli and Laura Iaffaldano contributed equally to the paper.

\section{Acknowledgments}

The authors thank Jean Ann Gilder (Scientific Communication SRL, Naples, Italy) for revising and editing the manuscript and Vittorio Lucignano, CEINGE-Biotecnologie Avanzate, for technical assistance related to graphics. They also acknowledge grants from CEINGE Regione Campania (DGRC 1901/2009), IRCCS SDN, Ministry of Health, POR Campania FSE 2007-2013-CUP B25B09000050007, Project CREME, and Project PON02_00619_3461281.

\section{References}

[1] N. Esser, S. Legrand-Poels, J. Piette, A. J. Scheen, and N. Paquot, "Inflammation as a link between obesity, metabolic syndrome and type 2 diabetes," Diabetes Research and Clinical Practice, vol. 105, no. 2, pp. 141-150, 2014.

[2] F. Favretti, G. Segato, D. Ashton et al., "Laparoscopic adjustable gastric banding in 1,791 consecutive obese patients: 12-year results," Obesity Surgery, vol. 17, no. 2, pp. 168-175, 2007.

[3] L. Iaffaldano, C. Nardelli, V. Pilone et al., "Laparoscopic adjustable gastric banding reduces subcutaneous adipose tissue and blood inflammation in nondiabetic morbidly obese individuals," Obesity Surgery, vol. 24, no. 12, pp. 2161-2168, 2014.

[4] W. T. Garvey, J. I. Mechanick, E. M. Brett et al., "American Association of Clinical Endocrinologists and American College of Endocrinology clinical practice guidelines for comprehensive medical care of patients with obesity-executive summary," Endocrine Practice, vol. 22, supplement 3, pp. 1-203, 2016.

[5] S. Vienberg, J. Geiger, S. Madsen, and L. T. Dalgaard, "MicroRNAs in metabolism," Acta Physiologica, 2016.

[6] R. Martinelli, C. Nardelli, V. Pilone et al., "MiR-519d overexpression Is associated with human obesity," Obesity, vol. 18, no. 11, pp. 2170-2176, 2010.

[7] V. Capobianco, C. Nardelli, M. Ferrigno et al., "MiRNA and protein expression profiles of visceral adipose tissue reveal miR-141/YWHAG and miR-520e/RAB11A as two potential miRNA/protein target pairs associated with severe obesity," Journal of Proteome Research, vol. 11, no. 6, pp. 3358-3369, 2012.

[8] Y. Peng, H. Xiang, C. Chen et al., "MiR-224 impairs adipocyte early differentiation and regulates fatty acid metabolism," International Journal of Biochemistry and Cell Biology, vol. 45, no. 8, pp. 1585-1593, 2013.
[9] Y. J. Kim, S. H. Hwang, H. H. Cho, K. K. Shin, Y. C. Bae, and J. S. Jung, "MicroRNA 21 regulates the proliferation of human adipose tissue-derived mesenchymal stem cells and high-fat diet-induced obesity alters microRNA 21 expression in white adipose tissues," Journal of Cellular Physiology, vol. 227, no. 1, pp. 183-193, 2012.

[10] N. Arias, L. Aguirre, A. Fernández-Quintela et al., "MicroRNAs involved in the browning process of adipocytes," Journal of Physiology and Biochemistry, vol. 72, no. 3, pp. 509-521, 2016.

[11] T. Fu, S. Seok, S. Choi et al., "MicroRNA 34a inhibits beige and brown fat formation in obesity in part by suppressing adipocyte fibroblast growth factor 21 signaling and SIRT1 function," Molecular and Cellular Biology, vol. 34, no. 22, pp. 4130-4142, 2014.

[12] J. Yu, Y. Chen, L. Qin et al., "Effect of miR-205 on 3T3L1 preadipocyte differentiation through targeting to glycogen synthase kinase 3 beta," Biotechnology Letters, vol. 36, no. 6, pp. 1233-1243, 2014.

[13] P. Arner and A. Kulyté, "MicroRNA regulatory networks in human adipose tissue and obesity," Nature Reviews Endocrinology, vol. 11, no. 5, pp. 276-288, 2015.

[14] A. Masotti, A. Baldassarre, M. Fabrizi et al., "Oral glucose tolerance test unravels circulating miRNAs associated with insulin resistance in obese preschoolers," Pediatric Obesity, 2016.

[15] M. C. Sugden, P. W. Caton, and M. J. Holness, "PPAR control: it's SIRTainly as easy as PGC," Journal of Endocrinology, vol. 204, no. 2, pp. 93-104, 2010.

[16] D. Portius, C. Sobolewski, and M. Foti, "MicroRNAs-dependent regulation of PPARs in metabolic diseases and cancers," PPAR Research, vol. 2017, Article ID 7058424, 19 pages, 2017.

[17] A. Wanet, A. Tacheny, T. Arnould, and P. Renard, "MiR-212/132 expression and functions: within and beyond the neuronal compartment," Nucleic Acids Research, vol. 40, no. 11, pp. 47424753, 2012.

[18] K. Schuch, B. Wanko, K. Ambroz et al., "Osteopontin affects macrophage polarization promoting endocytic but not inflammatory properties," Obesity, vol. 24, no. 7, pp. 1489-1498, 2016.

[19] M. Estep, D. Armistead, N. Hossain et al., "Differential expression of miRNAs in the visceral adipose tissue of patients with non-alcoholic fatty liver disease," Alimentary Pharmacology and Therapeutics, vol. 32, no. 3, pp. 487-497, 2010.

[20] J. Xiao, Y. Bei, J. Liu et al., "miR-212 downregulation contributes to the protective effect of exercise against non-alcoholic fatty liver via targeting FGF-21," Journal of Cellular and Molecular Medicine, vol. 20, no. 2, pp. 204-216, 2016.

[21] M. Á. Gómez-Sámano, M. Grajales-Gómez, J. M. ZuarthVázquez et al., "Fibroblast growth factor 21 and its novel association with oxidative stress," Redox Biology, vol. 11, pp. 335341, 2017. 


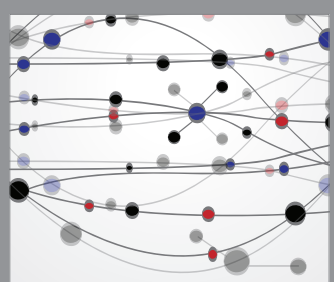

The Scientific World Journal
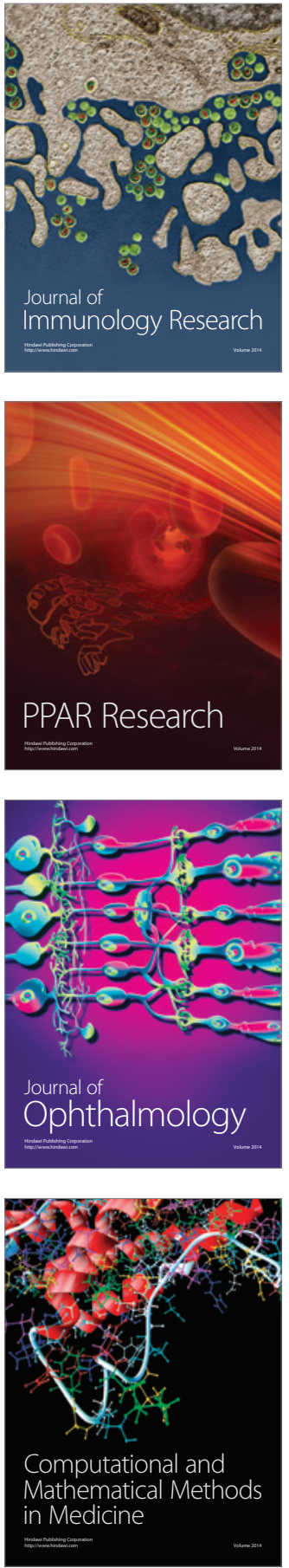

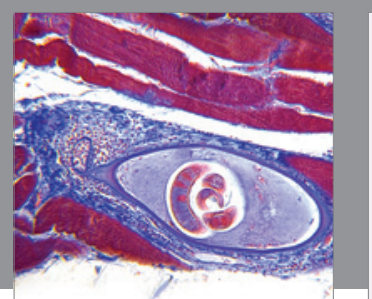

Gastroenterology Research and Practice
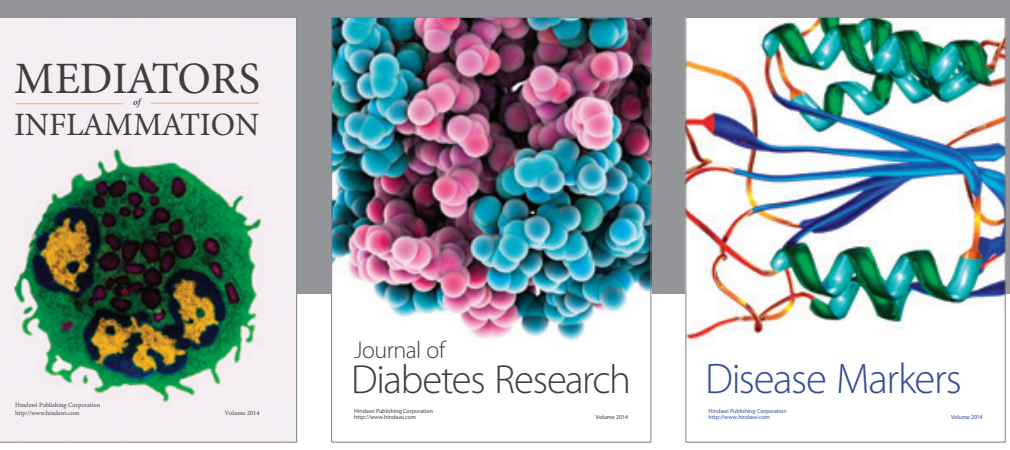

Disease Markers

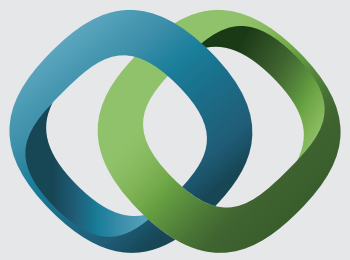

\section{Hindawi}

Submit your manuscripts at

https://www.hindawi.com
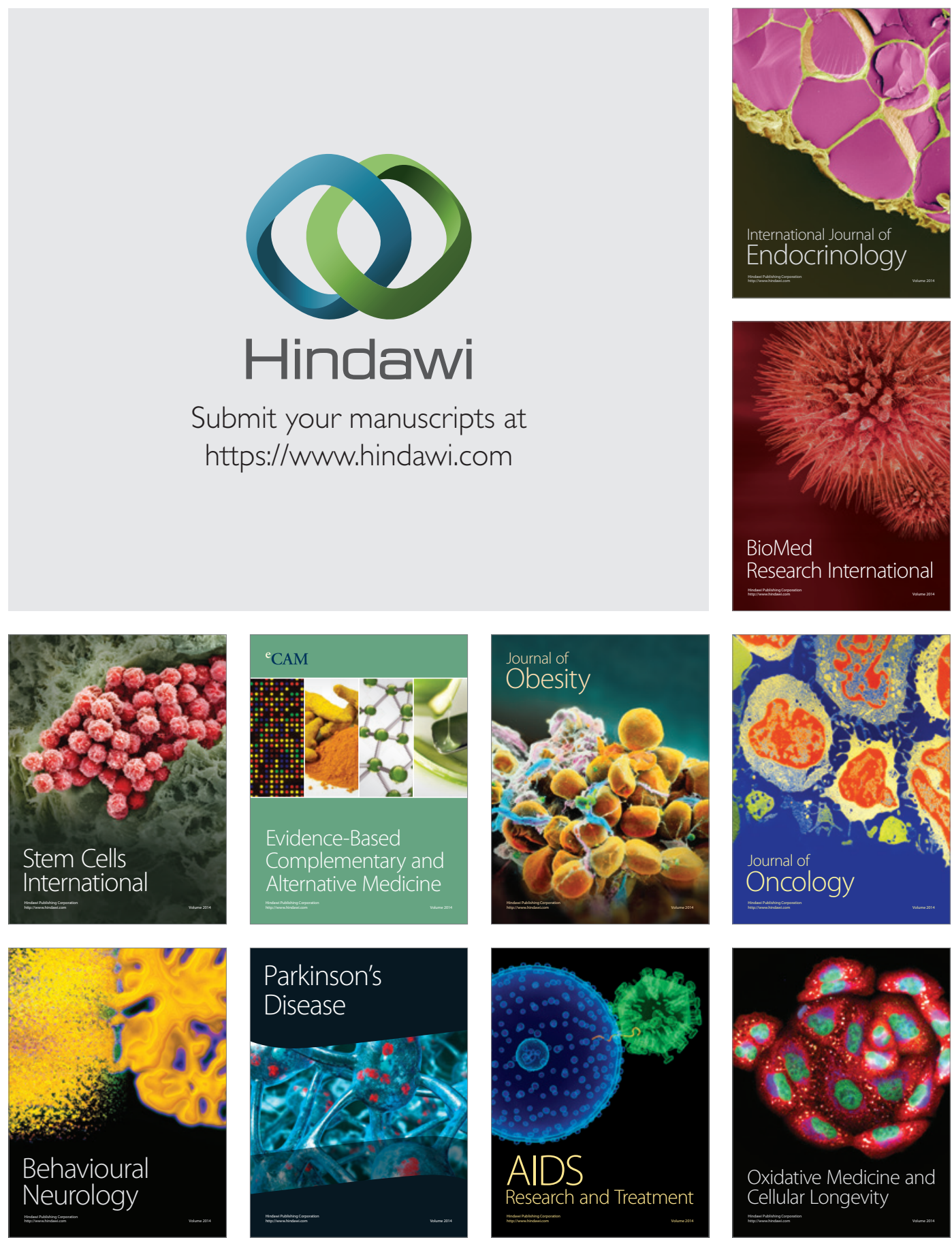